\title{
Single Molecule Protein Detection with Attomolar Sensitivity Using Droplet Digital Enzyme- Linked Immunosorbent Assay
}

Limor Cohen $^{\dagger 1,2,3}$, Naiwen Cui ${ }^{\dagger 4}$, Yamei Cai ${ }^{4}$, Padric M. Garden ${ }^{1,2}$, Xiang Li $^{1,2}$, David A. Weitz ${ }^{1,4}$, David R. Walt ${ }^{1,2,3}$

1. Wyss Institute for Biologically Inspired Engineering, Harvard University, Boston, MA 02115, USA.

2. Department of Pathology, Brigham and Women's Hospital, Harvard Medical School, Boston, MA 02115, USA.

3. Department of Chemical Biology, Harvard University, Boston, MA 02115, USA.

4. School of Engineering and Applied Sciences, Harvard University, Cambridge, MA 02138, USA

$\uparrow$ Co-first authors

*Corresponding Author: David R. Walt, Ph.D., Department of Pathology, Brigham and Women's Hospital, 60 Fenwood Road, Boston MA 02115. dwalt@bwh.harvard.edu

Figure S1: Image processing of droplets using the brightfield layer.

Figure S2: Image processing of bead layer.

Figure S3: Integration of the three image layers (droplets, beads, and fluorescent intensity of the enzyme's substrate).

Figure S4: Background Correction.

Figure S5: Aqueous bead solution inside the tubing.

Figure S6: Histograms of IFN $\gamma$ and IL-2 at various concentrations.

Figure S7: ddELISA LOD based on percentage of beads analyzed.

Table S1: $\mathrm{R}^{2}$ values of the Simoa and ddELISA calibration curves.

Theoretical Calculations 

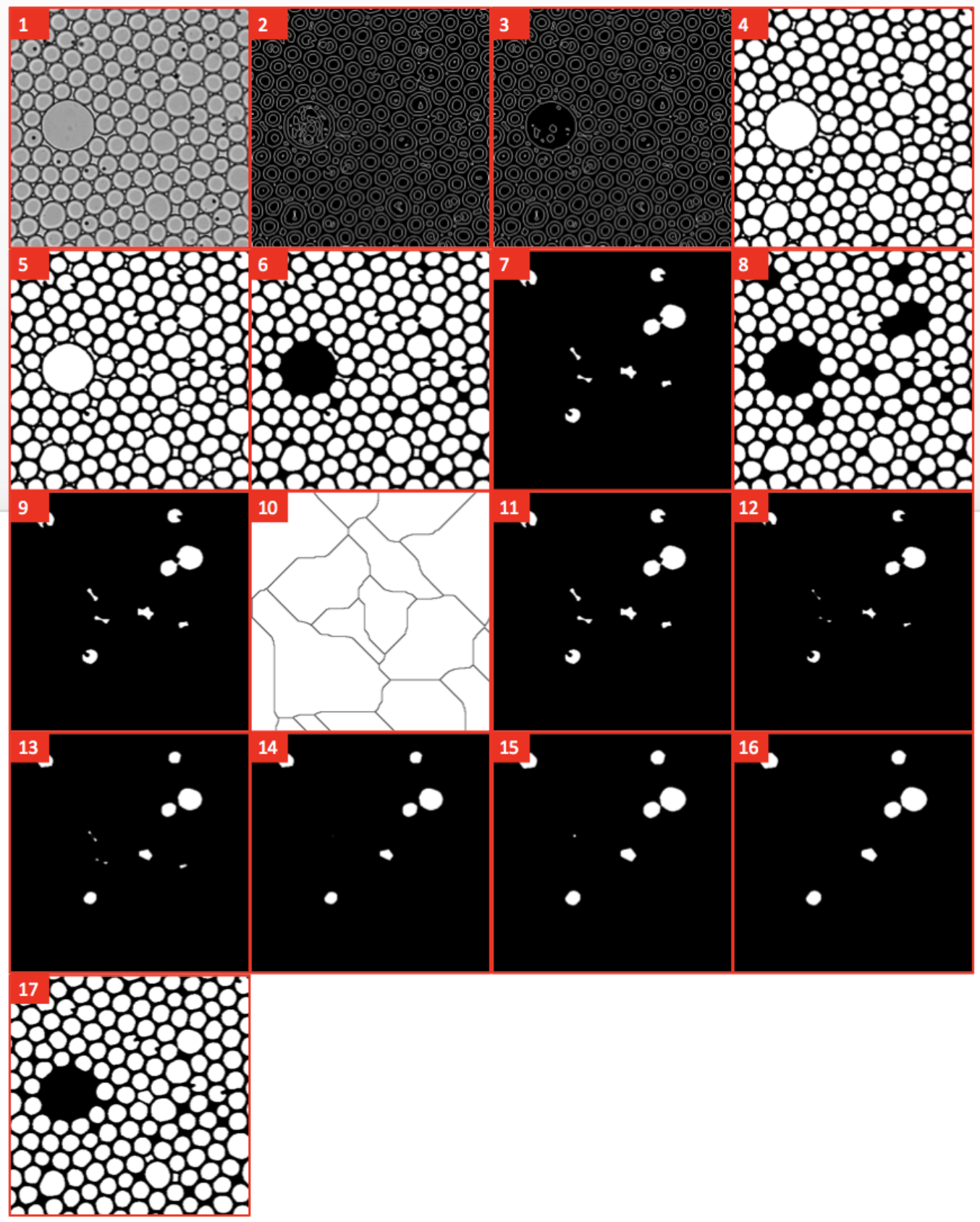

Figure S1: Image processing of droplets using the brightfield layer.

Matlab code for processing droplet layer:

1. BFADJUST = imadjust(imcrop(BF,[xLoc, yLoc, 2048, 2048]));

2. BFEdges = edge(BFADJUST,'log',0,3);

3. BFEdges $=$ bwareafilt(BFEdges, $[5$ 300]);

4. bw_a $=$ bw; bw_a $(1,:)=1 ; b w \_a(:, 1)=1$;

bw_a_filled =imfill(bw_a,'holes');

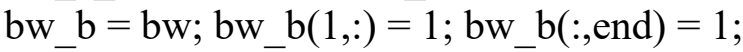

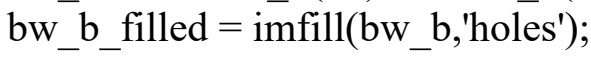


bw_c $=$ bw; bw_c(end,:) $=1$; bw_c $(:$, end $)=1$;

bw_c_filled $=$ imfill $($ bw_c,'holes');

bw_d = bw; bw_d(end,:) $=1$; bw_d $(:, 1)=1$;

bw_d_filled $=$ imfill $\left(\mathrm{bw}_{-} \mathrm{d}\right.$, 'holes' $)$;

5. BW_temp $=$ imclearborder $(\mathrm{bw}$ _a filled $\mid \mathrm{bw}$ _b_filled $\mid \mathrm{bw}$ _c_filled $\mid \mathrm{bw}$ _d_filled $)$;

6. $\mathrm{BW} \overline{2}=$ bwareafilt(BW_temp, $[1003000])$;

7. $\mathrm{BW} 3=$ bwpropfilt(BW2,'solidity',[0,0.9]);

8. $\mathrm{BW} 4=\mathrm{BW} 2$;

$\mathrm{BW} 4(\mathrm{BW})=0$;

9. $\mathrm{BW} 3$ = bwpropfilt(BW2,'solidity',[0,0.9]);

10. $\mathrm{D}=-$ bwdist $(\sim \mathrm{BW} 3)$;

mask $=$ imextendedmin $(\mathrm{D}, 2)$;

D2 = imimposemin(D,mask);

Ld2 = watershed(D2);

11. $\mathrm{BW} 3(\mathrm{Ld} 2==0)=0$;

12. $\mathrm{BW} 3$ = imerode $(\mathrm{BW} 3$, strel('disk',2));

13. $\mathrm{BW} 3$ = bwconvhull(BW3,'objects',8);

14. BW3 = bwpropfilt(BW3,'Eccentricity', $[0,0.75])$;

15. $\mathrm{BW} 3$ = imdilate $(\mathrm{BW} 3$,strel('disk',2));

16. BW3 = bwareafilt(BW3,[50 3000]);

17. $\mathrm{BW}=\mathrm{BW} 4 \mid \mathrm{BW} 3$; 


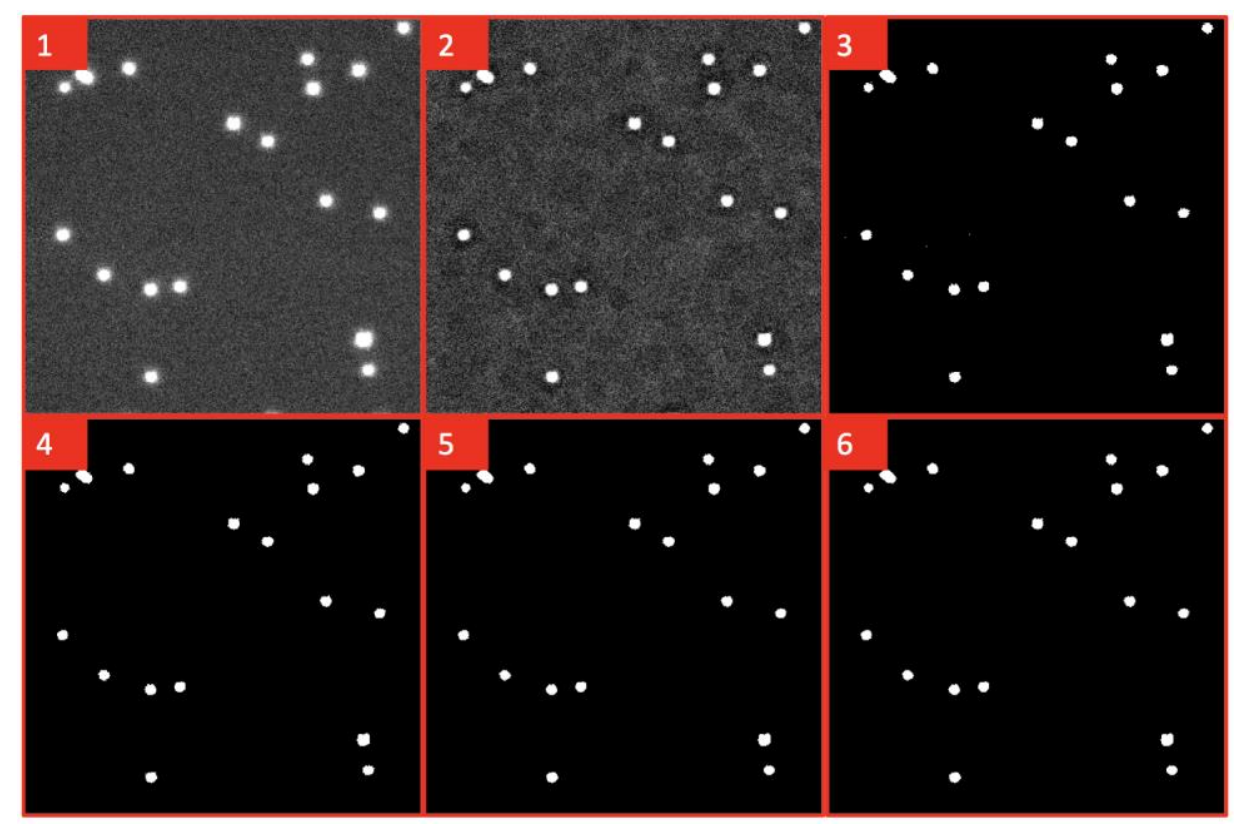

Figure S2: Image processing of bead layer.

Matlab code for processing bead layer:

1. Original image

2. BF_TH = imadjust(imtophat(fullBDimage,strel('disk',8)));

3. $\mathrm{BIN}=$ imbinarize $\left(\mathrm{BF}_{-} \mathrm{TH}, .8\right)$;

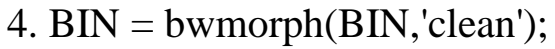

5. $\mathrm{BIN}=$ imopen $(\mathrm{BIN}$, strel('disk',1));

6. $\mathrm{BW}\{$ counter $\}=$ imclearborder(imcrop(BIN,[xLoc, yLoc, 2048, 2048])); 


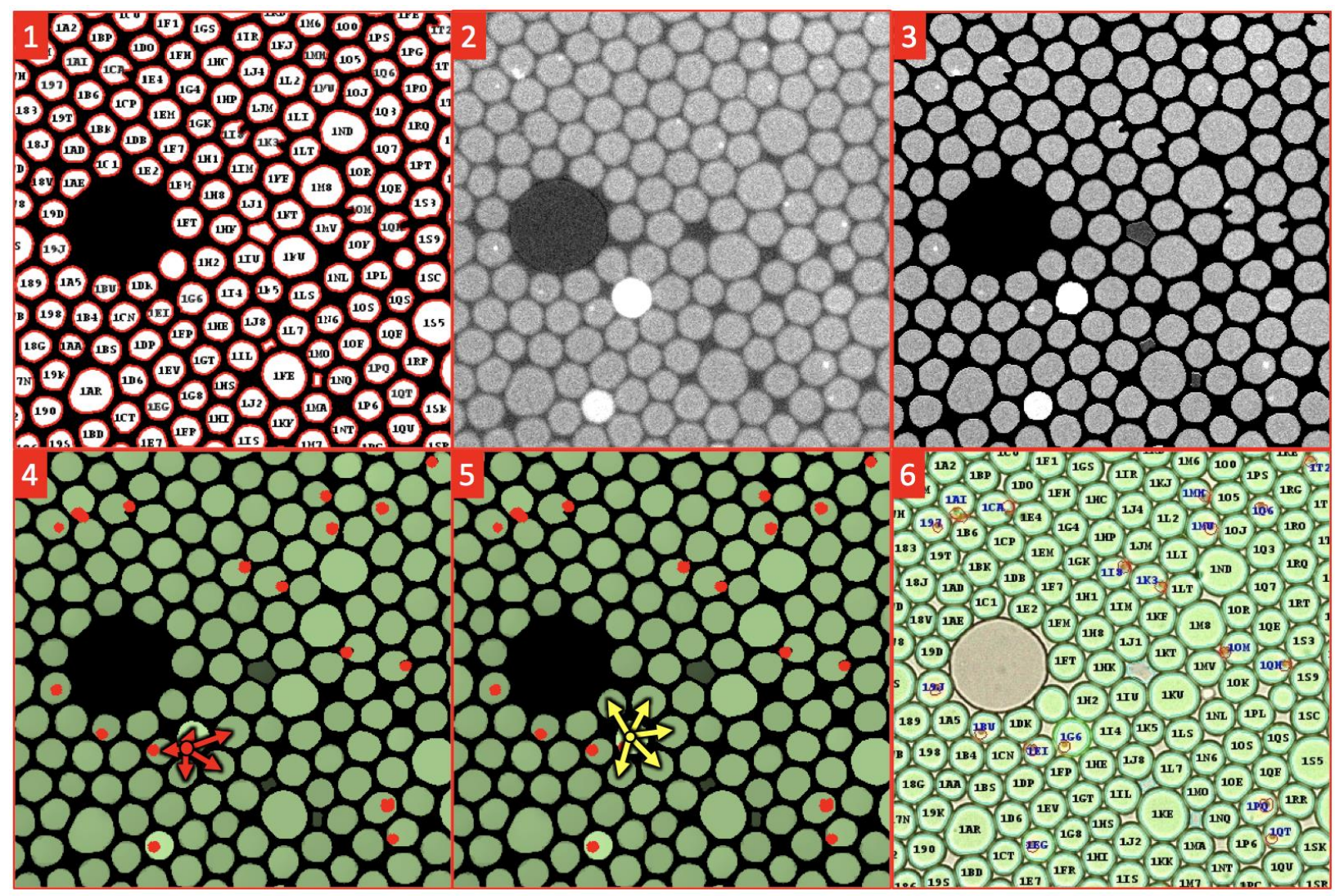

Figure S3: Integration of the three images (droplets, beads, and fluorescent intensity of the enzyme's substrate). After the droplets are identified using the brightfield image, each droplet is labeled with a unique identifier (1). Then, the unprocessed image of the fluorescent intensity of the enzyme's substrate (2) is overlaid with the processed brightfield image (3). Each droplet is then assigned an intensity value using the median intensity in the droplet. Then, beads are assigned to each droplet by first detecting the dye-encoded beads using the bead fluorescent image and then assigning each bead to a droplet with the most overlap (4). After all beads are assigned to a droplet, the five nearest droplet neighbors without a bead are identified and the median intensity of these five droplets is obtained. This median intensity is then subtracted from the intensity of the beadcontaining droplet to give an intensity value that is corrected for the local background (5). Droplets with less than four neighbors within 1.5 times the diameter of the bead-containing droplet are excluded. Validation images are then produced (6). 


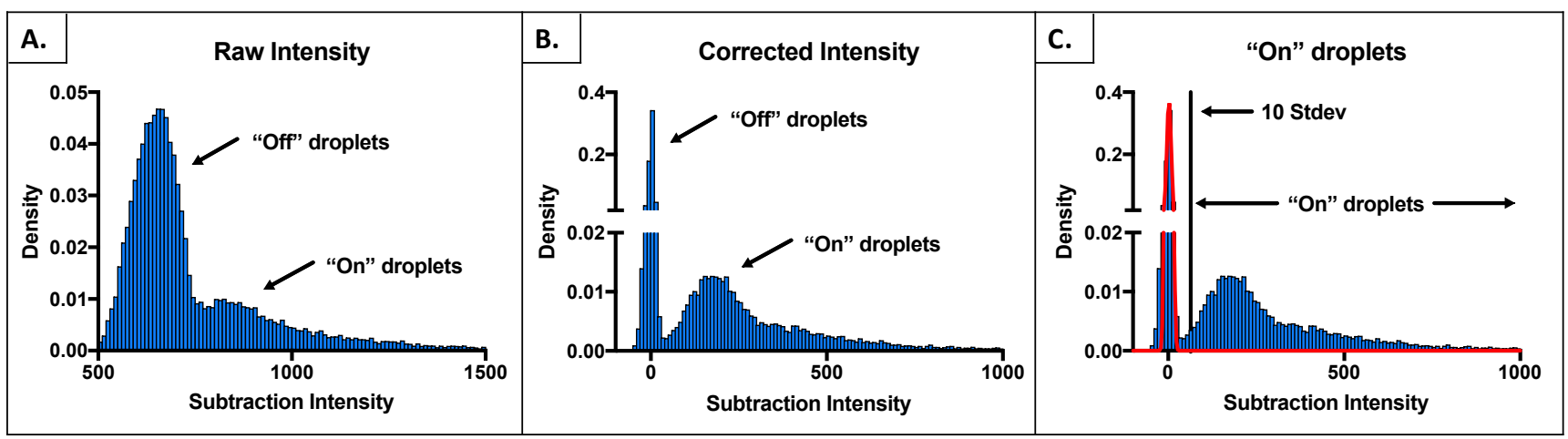

Figure S4: Background Correction. Example of background correction for $10 \mathrm{fM} \mathrm{IFN} \gamma$. A. Raw intensity of the "on" and "off" droplets. B. Corrected intensity of the "on" and "off" droplets. C. Determination of "on" droplets by modeling a Gaussian distribution and using ten standard deviations above the mean as the cutoff. 


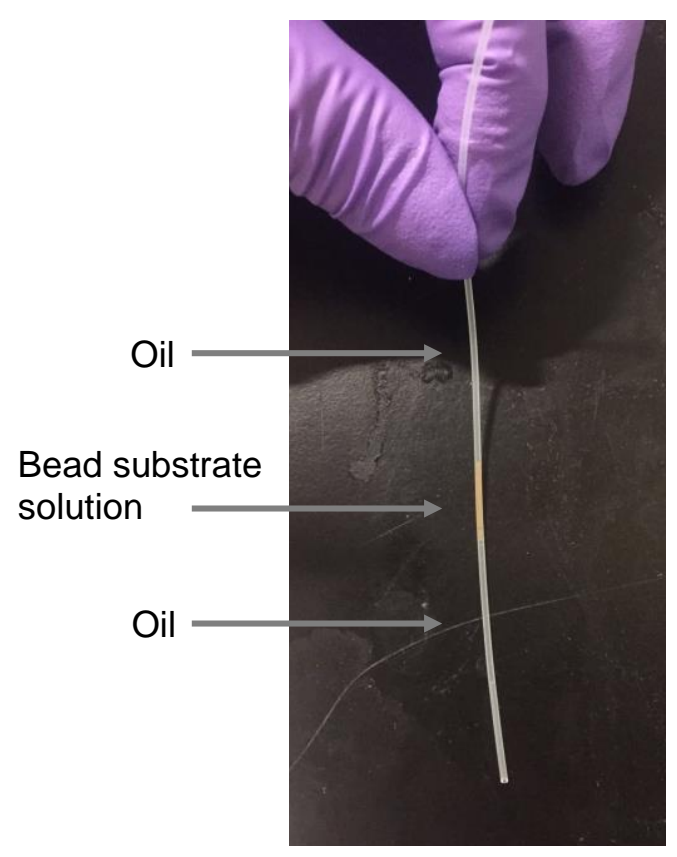

Figure S5: Aqueous bead solution inside the tubing. It is difficult to ensure droplet stability from start of droplet generation due to the low input volume $(2 \mu \mathrm{l})$ of the bead-substrate solution, while still generating many droplets per second. To solve this issue, we added oil before loading the aqueous bead solution into the tubing such that the oil will be injected first into the channel and stabilize the system prior to droplet generation. 

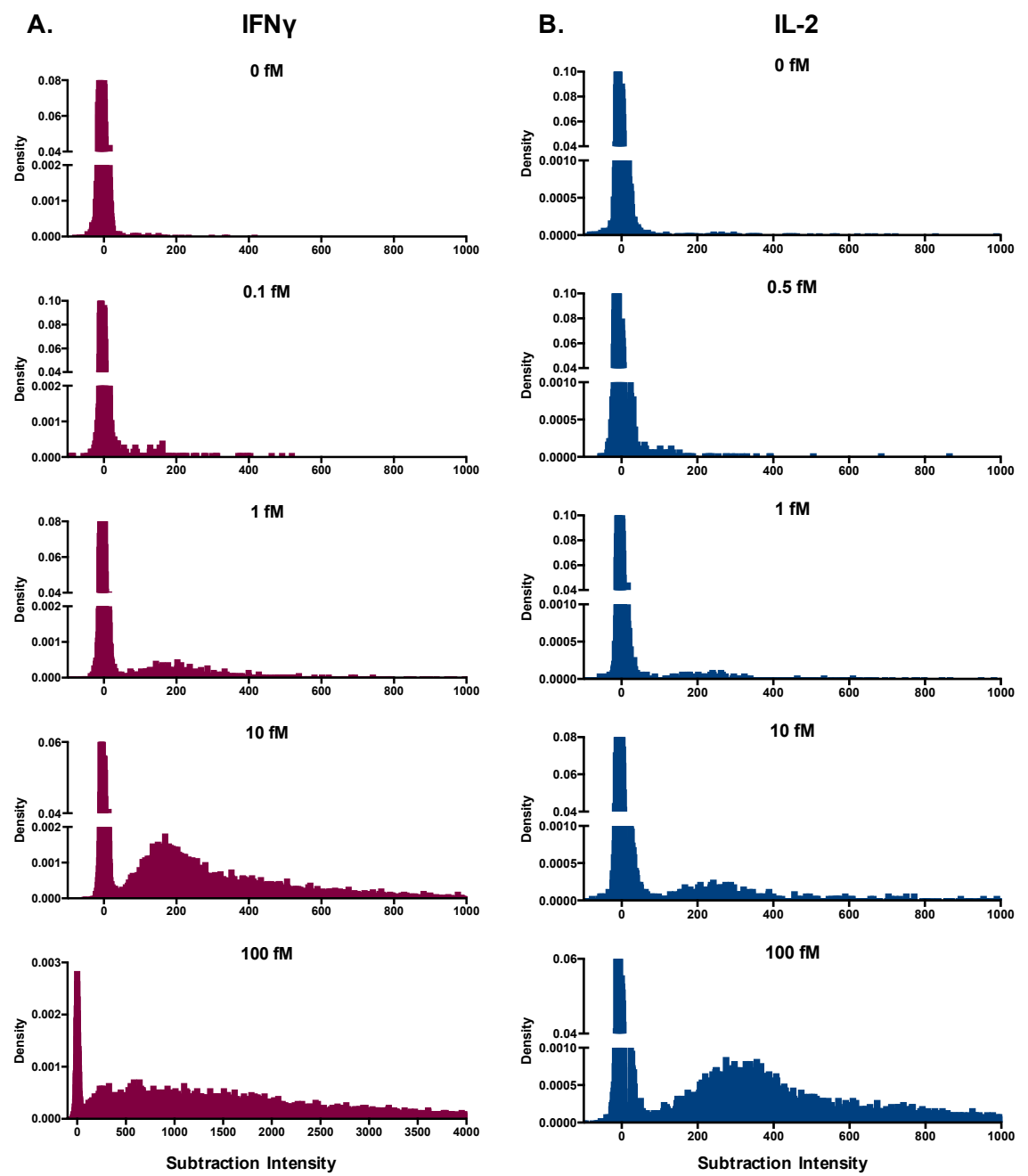

Figure S6: Histograms of IFN $\gamma$ and IL-2 at various concentrations. Histograms show the corrected signal intensity of each droplet containing a bead across various concentrations for IFN $\gamma$ (left) and IL-2 (right). 
IL-2

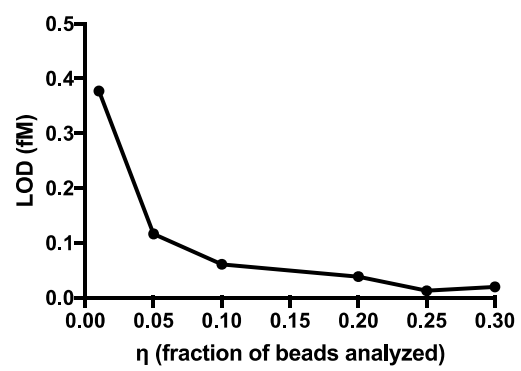

IFNy

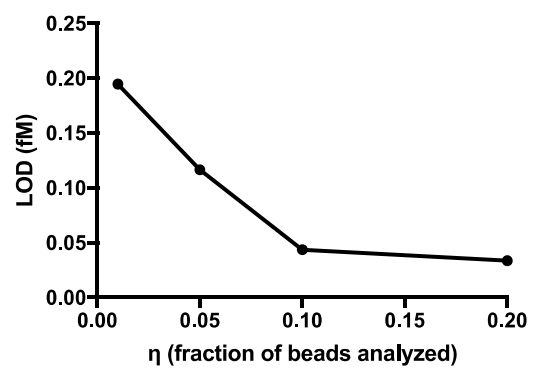

ORF1

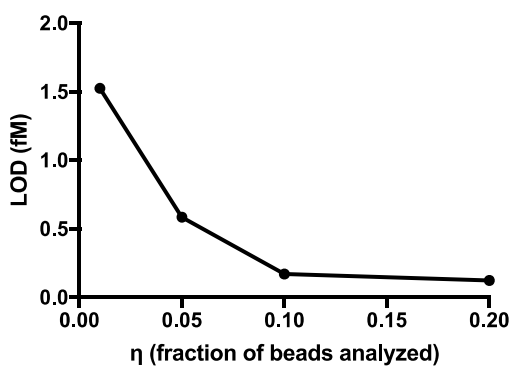

Figure S7: ddELISA LOD based on percentage of beads analyzed. For each biomarker, a subset of beads was randomly selected and analyzed. The LOD was then calculated. 


\begin{tabular}{|l|l|l|}
\hline Target & ddELISA & Simoa \\
\hline IFN $\gamma$ & 0.995 & 0.995 \\
\hline IL-2 & 0.954 & 0.998 \\
\hline ORF1 & 0.939 & 0.993 \\
\hline
\end{tabular}

Table S1: $\mathbf{R}^{\mathbf{2}}$ values of the Simoa and ddELISA calibration curves. Calibrators were measured in 3-5 replicates and the curve was fitted using a 4PL fit. 


\section{Theoretical Calculations}

The process of digital ELISA can be described by three sequential binding steps: (1) the capture antibody $(\mathrm{cAb})$ binds to the target protein analyte $(\mathrm{S})$, forming complex 1, (2) complex 1 binds to the detection antibody (dAb), forming complex 2, and (3) complex 2 binds to $S \beta G$, forming complex 3. Finally, the amount of complex 3 is measured using a digital readout system.

Step 1: $\quad c A b+S \stackrel{k_{d 1}}{\longrightarrow}$ complex 1

Step 2: $\quad$ complex $\# 1+d A b \stackrel{k_{d 2}}{\rightarrow}$ complex 2

Step 3: $\quad$ complex \#2+SBG $\stackrel{k_{d 3}}{\longrightarrow}$ complex 3

The concentrations of complexes 1-3 can be calculated based on the equilibrium of each step, assuming maximum reaction efficiency is reached.

capture + ligand $\rightarrow$ complex

$[\text { capture }]_{\text {total }}=[$ capture $]+[$ complex $]$

$[\text { ligand }]_{\text {total }}=[$ ligand $]+[$ complex $]$

$K_{D}=\frac{[\text { capture }][\text { ligand }]}{[\text { complex }]}$

Substituting equations (5) and (6) into equation (7):

$K_{D}=\frac{\left\{[\text { capture }]_{\text {total }}-[\text { complex }]\right\}\left\{[\text { ligand }]_{\text {total }}-[\text { complex }]\right\}}{[\text { complex }]}$

Calculating [complex]:

$[$ complex $]=0.5\left\{K_{D}+[\text { capture }]_{\text {total }}+[\text { ligand }]_{\text {total }}-\right.$

$\left.\sqrt{\left(K_{D}+[\text { capture }]_{\text {total }}+[\text { ligand }]_{\text {total }}\right)^{2}-4[\text { capture }]_{\text {total }}[\text { ligand }]_{\text {total }}}\right\}$

For each step (1-3), the concentration of complexes 1-3 can be determined using equation (9) by substituting the values of [capture $]_{\text {total, }}$ [ligand] $]_{\text {total }}$, and $K_{D}$ :

For step 1:

For step 2:

$$
\begin{gathered}
{[\text { capture }]_{\text {total }}=[\mathrm{cAb}]_{\text {total }}=[\text { beads }] * 250,000} \\
{[\text { ligand }]_{\text {total }}=[S]_{\text {total }}} \\
K_{D}=k_{d 1}
\end{gathered}
$$

$$
\begin{gathered}
{[\text { capture }]_{\text {total }}=[\text { complex } 1]} \\
{[\text { ligand }]_{\text {total }}=[\text { dAb }]_{\text {total }}} \\
K_{D}=k_{d 2}
\end{gathered}
$$


For step 3:

$$
\begin{gathered}
{[\text { capture }]_{\text {total }}=[\text { complex } 2]} \\
{[\text { ligand }]_{\text {total }}=[S B G]_{\text {total }}} \\
K_{D}=k_{d 3}
\end{gathered}
$$

We solve for [complex 3] with [S $]_{\text {total }}$ as a variable. The following assumptions are made: there are 100,000 beads, in which each bead has 250,000 antibody molecules coated on its surface. The reaction volumes are $100 \mu \mathrm{l}$, the detector antibody and $\mathrm{S} \beta \mathrm{G}$ concentrations are $0.3 \mu \mathrm{g} / \mathrm{mL}$ and 50 $\mathrm{pM}$, respectively, and $k_{d l}$ is equal to $k_{d 2}$. Since digital ELISA is limited by the Poisson noise, the boundary condition for the LOD is:

Poisson noise limited LOD $=\left\{[\text { complex \#3] }[\text { volume }] * \eta\}^{-0.5} \geq 10 \%\right.$

Where $\eta$ is the bead loading efficiency and the Poisson noise limited LOD is defined as $[S]_{\text {total }}$ that meets the above boundary condition (14). 